\title{
Executive function is necessary for the regulation of the stepping activity when stepping in place in older adults
}

\author{
Christopher Dalton $^{1} \cdot \operatorname{Ria} \operatorname{Sciadas}^{1} \cdot$ Julie $^{\text {Nantel }^{1}}$
}

Received: 30 October 2015/Accepted: 3 November 2015/Published online: 25 November 2015

(C) The Author(s) 2015. This article is published with open access at Springerlink.com

\begin{abstract}
To determine the effect of age on stepping performance and to compare the cognitive demand required to regulate repetitive stepping between older and younger adults while performing a stepping in place task (SIP). Fourteen younger $(25.4 \pm 6.5)$ and 15 older adults $(71.0 \pm 9.0)$ participated in this study. They performed a seated category fluency task and Stroop test, followed by a 60 s SIP task. Following this, both the cognitive and motor tasks were performed simultaneously. We assessed cognitive performance, SIP cycle duration, asymmetry, and arrhythmicity. Compared to younger adults, older adults had larger SIP arrhythmicity both as a single task and when combined with the Category $(p<0.001)$ and Stroop $(p<0.01)$ tasks. Older adults also had larger arrhythmicity when dual tasking compared to SIP alone $(p<0.001)$. Older adults showed greater SIP asymmetry when combined with Category $(p=0.006)$ and Stroop $(p=0.06)$ tasks. Finally, they had lower cognitive performance than younger adults in both single and dual tasks $(p<0.01)$. Age and type of cognitive task performed with the motor task affected different components of stepping. While SIP arrhythmicity was larger for all conditions in older compared to younger adults, cycle duration was not different, and asymmetry tended to be larger during SIP when paired with a verbal fluency task. SIP does not require a high level of control for dynamic stability, therefore demonstrating that higher-level executive function is necessary for the regulation of stepping activity independently of the
\end{abstract}

Julie Nantel

jnantel@uottawa.ca

1 Faculty of Health Sciences, School of Human Kinetics, Ottawa University, 125 rue Université, Pavillon Montpetit, MNT 353, Ottawa K1N 6N5, Canada regulation of postural balance. Furthermore, older adults may lack the cognitive resources needed to adequately regulate stepping activity while performing a cognitive task relying on the executive function.

Keywords Rhythmicity · Symmetry · Executive function · Older adults · Stepping · Gait

\section{Background}

Gait is considered a highly complex motor task, as postural balance must be regulated to achieve safe forward progression while also coordinating symmetrical actions of both lower limbs. Normal gait pattern requires that movements of the legs be similar in amplitude and timing [1-3]. However, healthy individuals walk with some spatial and temporal variability [1], which may reflect the flexibility and adaptability of the motor system in the regulation of the gait pattern [4]. Others have suggested that some gait characteristics such as stride-to-stride variability should be kept low to maintain gait stability [5, 6]. Supporting this suggestion is the notion that gait variability and asymmetry tend to increase with advancing age and in the presence of neurological deficits (e.g. Parkinson's disease) [7-9] and have been associated with a higher risk for falls in these populations [2, 10-14].

In younger adults, gait demands only minimal attention [15-17]. However, increasing evidence shows that gait regulation requires higher cognitive control, especially in older adults [18-20]. With advancing age, the ability to coordinate the action of each leg relative to the other becomes increasingly challenging, compromising gait symmetry, coordination, and ultimately reducing postural balance and increasing the risks for falls [17, 21]. 
Therefore, gait in older adults may not be as automatically regulated as it is in younger adults and may require more active control [17, 22, 23].

Dual-task paradigms have frequently been used to determine the cognitive processes involved in gait and often lead to a decrease in gait performance [15, 23-25] and an increased fall risk [17, 26]. Stride-to-stride variability has been found to be a reliable marker of gait regulation, where greater stride-to-stride variability represents poorer gait control as can be seen when two simultaneous tasks compete for one's limited available attentional resources. Therefore, dual tasking involves the appropriate allocation and prioritization of attention between the two competing tasks $[15,23,24,27]$. Typically, as the complexity of a cognitive task increases, the majority of attentional resources are directed towards the postural task to maintain motor performance and minimize postural instability $[15,28]$. When the cognitive task is simple, the threat of postural instability is low, but as task difficulty increases, so does the postural threat until prioritization between the two tasks is required [15, 28]. As postural task complexity increases, older adults devote greater attention to the postural task compared to younger adults $[18,27-$ 29].

As opposed to focusing on the entirety of a typical gait pattern, stepping-in-place (SIP) is a cyclical task, which reduces gait to its simplest form, as it does not include forward progression. Therefore, it reduces the need to regulate postural balance, and allows primarily assessing the regulation of the stepping action. SIP has been validated [30] and used as a surrogate to gait to assess stepping variability in individuals with Parkinson's disease $[10,30]$ and subsequently in virtual reality protocols in PD $[31,32]$.

The purposes of the present study are to determine the effect of age on stepping variability and to compare the cognitive demand required to regulate repetitive stepping when performing a simple SIP task. More specifically, this study aims to determine the effects of two cognitive tasks relying on executive processes, the Category task and the Stroop Word and Color Test, to compare their impact on stepping variability between younger and older adults. Our hypothesis is that age will have an impact on stepping performance and that concurrently performing a cognitive task while SIP will have a greater effect in older adults compared to younger adults.

\section{Methods}

Convenience sample of 29 subjects: 14 healthy younger adults (mean 25.4, SD 6.5, min 20, $\max 42$ years, 11 women) and 15 healthy older adults (mean 71.0, SD 9.0, $\min 60, \max 81$ years, 10 women) participated in the study. Subjects were excluded if they reported previous surgeries (i.e. knee or hip replacement), impairment (i.e. knee or back pain) or conditions (i.e. Type 2 diabetes) that may interfere with gait and balance. None of the recruited participants were actually excluded as participants were screened during a telephonic interview performed prior to testing. Subjects were excluded if they reported previous surgeries and/or impairments (e.g. knee or back pain) that could result in proprioceptive alteration (e.g. knee or hip replacement), or any conditions such as Type 2 diabetes that could result in peripheral neuropathy and therefore interfere with gait and balance. Participants with uncorrected vision or vestibular problems were also excluded from the study. None of the recruited participants were actually excluded as they were screened during a telephonic interview performed prior to testing. Following this pre-screening, participants performed the Montreal Cognitive Assessment (MoCA) and were excluded if they presented with potential Mild Cognitive Impairment, i.e. if they had a score below 26. The study was approved by our Institutional Review Board. Motor task Participants were asked to stand upright with their arms along their sides and step in place at a comfortable pace for $60 \mathrm{~s} \mathrm{[30].} \mathrm{SIP} \mathrm{trials,} \mathrm{were} \mathrm{performed} \mathrm{on} \mathrm{two}$ force platforms recording at $200 \mathrm{~Hz}$ (Kistler, Winterthur, Switzerland). Different from Nantel et al. [30], SIP cycle duration, symmetry and rhythmicity were calculated for $55 \mathrm{~s}$, to account for the non-steady state of the first few steps. SIP cycle asymmetry $=100 \times \mid \ln ($ SSWT/LSWT $) \mid$, where SSWT and LSWT correspond to the leg with the shortest and longest mean swing time over the trials, respectively. SIP cycle rhythmicity represents the mean stride time coefficient of variation $(\mathrm{CV})$ of both legs. A large stride time CV indicates less rhythmic gait. SIP rhythmicity and symmetry were averaged over three trials. Swing and stride times were analyzed using the algorithm by Nantel et al. [30]. Force plate data were filtered with a zero-lag fourth-order Butterworth filter with a $12 \mathrm{~Hz}$ cut-off frequency.

Cognitive tasks The Category task and the Stroop Word and Color Test were performed while sitting (single task condition) and while SIP (dual task condition). The categories were presented on a monitor placed $1 \mathrm{~m}$ in front of the participant. The total length of the trial was $60 \mathrm{~s}$, and each trial comprised of four categories (15 s each). The task consisted of naming as many items as possible that fit the given category in $15 \mathrm{~s}$ (e.g. naming as many vegetables as possible). Errors (e.g. naming a fruit in the vegetable category) were subtracted from the total number of items and categories were randomized between the sitting and SIP conditions. The Stroop Word and Color Test consisted of two parts: Stroop A (words) and Stroop B (colors). During the first part (Stroop A), participants were 
presented with an $8 \times 10$ matrix with color names written in different colored "inks". Participants had $30 \mathrm{~s}$ to read the color names out loud regardless of the color of the "ink" (e.g. blue written in red = blue.). In the second part (Stroop B), a second matrix was presented again for $30 \mathrm{~s}$, and participants were instructed to recite the color of the letters independently of the written word (e.g. blue written in red $=$ red.). This task assesses the executive function by looking at the ability to appropriately allocate attentional resources and resolve conflict, unlike the category test, which involves mostly semantic memory and executive functions such as flexibility and inhibition [33]. Variables For the Category test, the total number of responses was the main variable while for the Stroop Word and Color Test, the main variables included the total number of words, total number of colors, as well as both the word-color absolute and relative differences.

\section{Statistics}

Two-Way Mixed Design Analyses of Variance (ANOVAs) were used to account for the differences between age groups (younger and older adults) and attention (sitting and SIP) for cognitive performance (Category and Stroop tests). Two-Way Mixed Design ANOVAs were also used to compared SIP cycle duration, symmetry and rhythmicity between groups and between conditions (single or dual tasking). Repeated measures ANOVAs were also used to determine the difference between conditions within each group. Statistical level of significance was set at $p<0.05$. All significant results were subjected to Bonferroni adjustment for multiple comparisons.

\section{Results}

\section{Motor performance}

For SIP arrhythmicity (Table 1), there was a significant main effect for tasks, $F(2,26)=3.685, p<0.001$. Pairwise comparisons revealed a statistical difference between SIP as a single task $F(1,27)=13.022, p=0.001$ and SIP combined with the Category task, $F(1,27)=2.638$, $p=0.001$, and the Stroop Word and Color Test, $F(1$, $27)=8.752, p=0.006$. There was also a main effect for groups, $F(1,27)=17.814, p<0.001$. Older adults had significantly larger SIP arrhythmicity compared to younger adults when performing the SIP task as single task as well as when performed in combination with both the Category $(p=0.001)$ and Stroop Word and Color Test $(p<0.01)$, Table 1. Within the older adult group, SIP arrhythmicity during single tasking was smaller than during the Category and the Stroop Word and Color Test $(p<0.001)$. Also, when comparing dual task conditions, SIP arrhythmicity was smaller during the Stroop Word and Color Test compared to the Category test $(p=0.02)$. No differences were seen within the younger adult group.

In SIP asymmetry, there was a main effect for tasks, $F(2,26)=3.869, \quad p=0.03$. Pairwise comparisons revealed a difference between SIP as a single task and SIP combined with the Category task $(p=0.03)$. Within the older adult group, asymmetry of SIP alone was smaller compared to SIP with the Category task $(p=0.006)$ and a trend was seen with the Stroop Word and Color Test $(p=0.06)$. No differences were seen within the younger adult group.

No main effects were seen when comparing SIP asymmetry between groups, $F(1,27)=3.503, p=0.072$. However, results showed a trend for statistical difference between groups in the Category task $(p=0.06)$. SIP stride duration in single or dual tasking was not significantly different between the groups or tasks.

\section{Cognitive performance}

In the Category task, the total number of items named showed a main effect for tasks, $F(1,27)=7.029$, $p=0.01$, as well as a main effect for groups $F(1$, $27)=15.962, p<0.001$. Older adults named fewer items compared to the younger group for both single tasking, $F(1,27)=8.169, p=0.008$, and dual tasking, $F(1$, $27)=20.499, p<0.001$. Within the older adult group, the total number of responses during the Category test was lower when SIP (mean 27.5, SD 4.9) compared to when seated (mean 30.8, SD 7.3, $p<0.05$ ). There were no differences between single (mean 37.3, SD 4.2) and dual (mean 35.8, SD 5.0) tasks in younger adults.

For the Stroop Word and Color Test, the number of words revealed a main effect for task, $F(1,27)=17.708$, $p<0.001$, as well as for groups, $F(1,27)=13.348$, $p=0.001$, Table 2. Pairwise comparisons revealed a difference between groups, with older adults identifying fewer items compared to younger adults when single tasking, $F(1,27)=7.162, p=0.01$, and when dual tasking, $F(1$, $27)=13.236, p=0.001$. Within the older adult group, fewer words were identified during dual tasking compared to single tasking $(p<0.01)$. No differences were seen between single and dual tasking conditions in younger adults.

The number of colors showed no main effect for task. However, there was a main effect for groups, $F(1$, $27)=57.245, p<0.001$. Pairwise comparisons revealed fewer colors identified by older adults compared to younger adults both in single, $F(1,27)=48.212$, $p<0.001$, and dual tasking conditions $F(1,27)=48.079$, $p<0.001$. 
Table 1 Asymmetry and arrythmicity (mean \pm SD) as a single task and combined with the Category task and the Stroop Color-Word Test

\begin{tabular}{|c|c|c|c|c|c|c|}
\hline & \multicolumn{3}{|c|}{ Young adults $(n=14)$} & \multicolumn{3}{|c|}{ Older adults $(n=15)$} \\
\hline & Single task & Category & Stroop & Single task & Category & Stroop \\
\hline Arryhthmicity (CV) & $2.63 \pm 0.61$ & $3.65 \pm 0.87$ & $3.53 \pm 0.60$ & $3.89 \pm 1.16^{\dagger}$ & $6.27 \pm 2.62^{\dagger *}$ & $5.20 \pm 2.04^{\dagger * \omega}$ \\
\hline Asymmetry & $4.13 \pm 1.56$ & $4.88 \pm 2.47$ & $4.73 \pm 2.83$ & $4.61 \pm 1.79$ & $6.93 \pm 3.16^{*}$ & $6.09 \pm 2.52$ \\
\hline Stepping in place cycle (s) & $1.15 \pm 0.14$ & $1.18 \pm 0.15$ & $1.17 \pm 0.14$ & $1.13 \pm 0.21$ & $1.18 \pm 0.23$ & $1.14 \pm 0.24$ \\
\hline
\end{tabular}

$\dagger$ Significantly different from young $p<0.01$

* Significantly different from single task $p<0.01$

${ }^{\omega}$ Significantly different from category task $p<0.05$

Table 2 Stroop Color-Word Test (mean \pm SD) while sitting (single task) and when combined with the SIP task

\begin{tabular}{|c|c|c|c|c|}
\hline & \multicolumn{2}{|c|}{ Young adults $(n=14)$} & \multicolumn{2}{|c|}{ Older adults $(n=15)$} \\
\hline & Single task & Dual task & Single task & Dual task \\
\hline Number of words & $66.9 \pm 9.6$ & $60.4 \pm 9.5$ & $56.7 \pm 10.8^{\dagger}$ & $47.1 \pm 10.1^{\dagger+}$ \\
\hline Number of colours & $34.4 \pm 4.2$ & $36.4 \pm 5.2$ & $19.1 \pm 7.2^{\dagger}$ & $19.3 \pm 7.7^{\dagger}$ \\
\hline Difference (words-colours) & $32.5 \pm 9.2$ & $24.1 \pm 7.8^{\ddagger}$ & $37.6 \pm 12.8$ & $27.8 \pm 8.6^{*}$ \\
\hline Colours/words (\%) & $52.1 \pm 8.0$ & $60.9 \pm 9.0^{*}$ & $34.7 \pm 13.7^{\dagger}$ & $40.9 \pm 13.9^{\dagger}$ \\
\hline
\end{tabular}

${ }^{\dagger}$ Significantly different from young $p<0.05$

* Significantly different from single task $p<0.05$
The Stroop Word and Color Test difference showed a main effect for task, $F(1,27)=16.390, p<0.001$. Both older $(p<0.001)$ and younger adults $(p=0.03)$ showed a larger word-colors difference in dual tasking compared to single tasking. The Stroop Word and Color Test difference ratio showed a main effect for task, $F(1,27)=9.453$, $p=0.005$, and a main effect for groups, $F(1$, $27)=27.956, p<0.001$. Pairwise comparisons showed smaller ratio in older adults compared to younger adults both in the single task, $F(1,27)=17.127, p<0.001$, and dual task $F(1,27)=20.656, p<0.001$. Within the older adults, the Stroop Word and Color Test difference ratio was larger in dual tasking $(0.41 \pm 0.03)$ compared to single tasking $(0.35 \pm 0.03), p<0.001$. Within the younger adults, the ratio was also larger in dual tasking $(0.61 \pm 0.03)$ compared to single tasking $(0.52 \pm 0.03)$, $p<0.001$.

\section{Discussion}

The main purposes of this study were to determine the effect of age on motor and cognitive performance and the impact of a two cognitive tasks relying on the executive function, the Category verbal fluency task and the Stroop Word and Color Test, on SIP regulation. Contrary to previous studies assessing the effect of age and dual tasking on gait variability, we chose the SIP task as it does not require control of the center of mass for forward gait progression and therefore allows for sole assessment of the stepping activity generation and regulation. As expected, our results showed that both motor and cognitive performances were affected by age. More importantly, we found that age and the type of cognitive task performed along with the motor task had an effect on different components of gait and cognitive performance. While arrhythmicity was larger in all conditions in older compared to younger adults, SIP cycle duration was not significantly different in any of the conditions. A trend toward larger asymmetry was found with older adults $(p=0.06)$, but only when SIP was combined with the Category task, not the Stroop.

The similar SIP cycle duration between groups and conditions is different from other studies which found slower gait speed in older adults especially when dual tasking. During normal walking, the progression of the center of mass within the margin of stability needs to be actively controlled by the central nervous system. Therefore, a reduction in speed when gait is combined with a cognitive task provides more time to adequately position the foot on the ground, thus preserving postural stability. Contrary to walking, SIP does not require a high level of regulation of dynamic stability, as the forward progression of the center of mass does not need to be controlled. Therefore, foot placement during SIP may require less 
active regulation than normal walking and consequently allow more of one's cognitive resources to be allocated to the cognitive performance. As gait velocity has been shown to decrease in both fallers $[34,35]$ and in individuals with cognitive impairments [36-38], it would have been interesting to determine if cycle duration would decrease in these populations despite the simplicity of the SIP task. However, a larger sample size would have been necessary to further divide our older adult group.

Previous studies have reported larger gait variability in older adults when performing a Category fluency task [29], but have shown no effect in younger adults [23, 29]. Recently, Walshe et al. [22] reported a larger negative effect of dual tasking on gait, but even more so when the cognitive task targeted the executive functions. In their study, gait in both young and older adults was affected, but with a larger effect in older adults. Altogether, these studies demonstrated that the generation of the gait pattern involves higher cortical regions [22, 23, 29]. The greater effect of dual tasking on older adults also suggests that as age progresses, cognitive resources may be inadequate to perform gait and a cognitive task simultaneously $[12,22]$. Overall, our results are in line with these previous studies, which demonstrate that age and dual tasking affect the generation and regulation of the SIP activity. However, SIP arrhythmicity was larger in older adults, independently of the cognitive task, while asymmetry was not affected until concomitant cognitive activity was performed. This suggests that age affects the ability to regulate stepping rhythmicity to a greater extent than it affects stepping asymmetry, and that both necessitate higher-level cognitive functions independently of the control of postural balance.

Overall, cognitive performance was affected by age both in single and dual tasking conditions, which highlights the age-related decline in executive processes performance [39]. Interestingly, both the Category task and Stroop A (words, congruent) showed a main effect for tasks while Stroop B (colours, incongruent) did not. This could be due to what was previously described by Bloem and collaborators [27] as the posture first strategy, whereby older adults could have been prioritizing motor performance over cognitive performance when the level of difficulty of the cognitive task was increased (incongruent vs. congruent). The positive aspect of such a strategy is that it reduces the risk of postural instability during the completion of the motor task. However, on the basis of the older adults' decreased performance in both the motor and cognitive task compared to younger adults, these strategies may not be entirely effective. As participants in the present study were healthy older adults, it is more likely that motor and cognitive performances in individuals with cognitive deficits would have been even more affected by the dual task paradigm [34-38].

The type of cognitive task chosen is important to consider when looking at its effect on gait, as it has been demonstrated that tasks involving executive function have a larger effect on gait compared to tasks used to divide attention (e.g. reciting the alphabet) [20, 22, 40]. Both cognitive tasks performed in this study are considered to rely mainly on the executive functions. However, specific characteristics of these tasks could explain motor and cognitive discrepancies between the tasks. While verbal fluency is largely attributed to the executive functions, it has been shown to be dependent on the visuospatial sketchpad, a subsystem of the working memory, and therefore, participants may use visual imagery to retrieve items from a category [41]. In our study, despite the fact that participants were asked to fixate on a monitor during the trial, the visualization strategy could have led to an internal focus of attention. This is important when considering that the Stroop Word and Color Test necessitates focusing "externally" on the monitor. Therefore, the monitor could have played the role of a visual anchor, stabilizing balance and reducing stepping asymmetry by a greater extent during the Stroop Word and Color Test than during the category task.

\section{Conclusions}

Age and the type of a cognitive task affected stepping characteristics differently. While SIP arrhythmicity was larger in all conditions in older adults compared to younger adults, SIP cycle duration was not significantly different and asymmetry showed a trend to be larger when a verbal fluency task was performed while stepping in place. This suggests that the regulation of the stepping activity relies more heavily on the executive function as age progresses and that the age related decline in these higher-level cognitive functions affects the ability to generate rhythmical stepping cycles. Considering that this study was conducted on healthy older adults, it would be interesting to assess stepping variability in older adults with cognitive decline and in individuals at high risk of falls.

Acknowledgments The study was approved by the University of Ottawa University Institutional Review Board. This work was supported by the Research Development Program, Faculty of Health Sciences, U. Ottawa (Julie Nantel) as well as by Undergraduate Research Opportunity Program, Faculty of Health Sciences, U. Ottawa (Ria Sciadas). We would like to thank Shaun Porter for his involvement in testing participants. 


\section{Compliance with ethical standards}

Conflict of interest The authors declare no conflict of interest.

Human and animal rights All procedures performed in studies involving human participants were in accordance with the ethical standards of the institutional and/or national research committee and with the 1964 Helsinki declaration and its later amendments or comparable ethical standards.

Informed consent Informed consent was obtained from all individual participants included in the study.

Open Access This article is distributed under the terms of the Creative Commons Attribution 4.0 International License (http://crea tivecommons.org/licenses/by/4.0/), which permits unrestricted use, distribution, and reproduction in any medium, provided you give appropriate credit to the original author(s) and the source, provide a link to the Creative Commons license, and indicate if changes were made.

\section{References}

1. Sadeghi H, Allard P, Prince F et al (2000) Symmetry and limb dominance in able-bodied gait: a review. Gait Posture 12:34-45

2. Yogev G, Plotnik M, Peretz C et al (2007) Gait asymmetry in patients with Parkinson's disease and elderly fallers: when does the bilateral coordination of gait require attention? Exp Brain Res 177:336-346

3. Forczek W, Staszkiewicz R (2012) An evaluation of symmetry in the lower limb joints during the able-bodied gait of women and men. J Hum Kinet 35:47-57

4. Krishnan V, Rosenblatt NJ, Latash ML et al (2013) The effects of age on stabilization of the mediolateral trajectory of the swing foot. Gait Posture 38:923-928

5. Beauchet O, Allali G, Annweiler C et al (2009) Gait variability among healthy adults: low and high stride-to-stride variability are both a reflection of gait stability. Gerontology 55:702-706

6. Hausdorff JM (2007) Gait dynamics, fractals and falls: finding meaning in the stride-to-stride fluctuations of human walking. Hum Mov Sci 26:555-589

7. Kline JE, Poggensee K, Ferris DP (2014) Your brain on speed: cognitive performance of a spatial working memory task is not affected by walking speed. Front Hum Neurosci 8:288. doi:10. 3389/fnhum.2014.00288

8. Nantel J, McDonald JC, Tan S et al (2012) Deficits in visuospatial processing contribute to quantitative measures of freezing of gait in Parkinson's disease. Neuroscience 221:151-156

9. Yogev G, Giladi N, Peretz C et al (2005) Dual tasking, gait rhythmicity, and Parkinson's disease: which aspects of gait are attention demanding? Eur J Neurosci 22:1248-1256

10. Nantel J, McDonal JC, Bronte-Stewart H (2014) The effect of medication and the role of postural instability in different components of freezing of gait (FOG). Parkinsonism Relat Disord 20:447-451

11. Toebes MJ, Hoozemans MJ, Furrer R et al (2012) Local dynamic stability and variability of gait are associated with fall history in elderly subjects. Gait Posture 36:527-531

12. Mirelman A, Herman T, Brozgol M et al (2012) Executive function and falls in older adults: new findings from a five-year prospective study link fall risk to cognition. PLoS One 7:e40297. doi:10.1371/journal.pone.0040297
13. Herman T, Mirelman A, Giladi N et al (2010) Executive control deficits as a prodrome to falls in healthy older adults: a prospective study linking thinking, walking and falling. J Gerontol A Biol Sci Med Sci 65:1086-1092

14. Springer S, Giladi N, Peretz C et al (2006) Dual-tasking effects on gait variability: the role of aging, falls, and executive function. Mov Disord 21:950-957

15. Beauchet O, Dubost V, Herrmann FR et al (2005) Stride-to-stride variability while backward counting among healthy young adults. J Neuroeng Rehabil 2:26. doi:10.1186/1743-0003-2-26

16. Malone LA, Bastian AJ (2010) Thinking about walking: effects of conscious correction versus distraction on locomotor adaptation. J Neurophysiol 103:1954-1962

17. Hausdorff JM, Schweiger A, Herman T et al (2008) Dual-task decrements in gait: contributing factors among healthy older adults. J Gerontol A Biol Sci Med Sci 63:1335-1343

18. Woollacott M, Shumway-Cook A (2002) Attention and the control of posture and gait: a review of an emerging area of research. Gait Posture 16:1-14

19. Yogev-Seligmann G, Hausdorff JM, Gailadi N (2008) The role of executive function and attention in gait. Mov Disord 23:329-342

20. Al-Yahya E, Dawes H, Smith L et al (2011) Cognitive motor interference while walking: a systematic review and meta-analysis. Neurosci Biobehav Rev 35:715-772

21. Plotnik M, Giladi N, Hausdorff JM (2007) A new measure for quantifying the bilateral coordination of human gait: effects of aging and Parkinson's disease. Exp Brain Res 181:561-570

22. Walshe EA, Patterson MR, Commins S et al (2015) Dual-task and electrophysiological markers of cognitive processing in older adult gait and fall-risk. Front Hum Neurosci 9:200. doi:10.3389/ fnhum.2015.00200

23. Dubost V, Kressig RW, Gonthier R, Herrmann FR et al (2006) Relationships between dual-task related changes in stride velocity and stride time variability in healthy older adults. Hum Mov Sci 25:372-382

24. Dubost V, Annweiler C, Aminian K et al (2008) Stride-to-stride variability while enumerating animal names among healthy young adults: result of stride velocity or effect of attention-demanding task? Gait Posture 27:138-143

25. Bock O, Beurskens R (2011) Age-related deficits in dual-task walking: the role of foot vision. Gait Posture 33:190-194

26. Ayoubi F, Launay CP, Kabeshova A et al (2014) The influence of fear of falling on gait variability: results from a large elderly population-based cross-sectional study. J Neuroeng Rehabil $11: 128$

27. Bloem BR, Valkenburg VV, Slabbekoorn M et al (2001) The multiple tasks test: development and normal strategies. Gait Posture 14:191-202

28. Yogev-Seligmann G, Hausdorff JM, Giladi N (2012) Do we always prioritize balance when walking? Towards an integrated model of task prioritization. Mov Disord 27:765-770

29. Yogev-Seligmann G, Rotem-Galili Y, Mirelman A et al (2010) How does explicit prioritization alter walking during dual-task performance? Effects of age and sex on gait speed and variability. Phys Ther 90:177-186

30. Nantel J, de Solages C, Bronte-Stewart H (2011) Repetitive stepping in place identifies and measures freezing episodes in subjects with Parkinson's disease. Gait Posture 34:329-333

31. Matar E, Shine JM, Naismith SL et al (2013) Using virtual reality to explore the role of conflict resolution and environmental salience in Freezing of Gait in Parkinson's disease. Parkinsonism Relat Disord 19:937-942

32. Shine JM, Matar E, Ward PB et al (2013) Exploring the cortical and subcortical functional magnetic resonance imaging changes 
associated with freezing in Parkinson's disease. Brain 136:1204-1215

33. Bastin C, Salmon E (2014) Early neuropsychological detection of Alzheimer's disease. Eur J Clin Nutr 68:1192-1199

34. Abellan van Kan G, Rolland Y, Andrieu S et al (2009) Gait speed at usual pace as a predictor of adverse outcomes in communitydwelling older people an International Academy on Nutrition and Aging (IANA) task force. J Nutr Health Aging 13:881-888

35. Verghese J, Holtzer R, Lipton RB et al (2009) Quantitative gait markers and incident fall risk in older adults. J Gerontol A Biol Sci Med Sci 64:896-901

36. Montero-Odasso M, Muir SW, Speechley M (2012) Dual-task complexity affects gait in people with mild cognitive impairment: the interplay between gait variability, dual tasking, and risk of falls. Arch Phys Med Rehab 93:293-299

37. Verghese J, Wang C, Lipton RB et al (2013) Motoric cognitive risk syndrome and the risk of dementia. J Gerontol A Biol Sci Med Sci 68:412-418
38. Beauchet O, Annweiler C, Montero-Odasso M et al (2012) Gait control: a specific subdomain of executive function? J Neuroeng Rehabil 9:12. doi:10.1186/1743-0003-9-12

39. DiGirolamo GJ, Kramer AF, Barad V et al (2011) General and task-specific frontal lobe recruitment in older adults during executive processes: a fMRI investigation of task-switching. NeuroReport 12:2065-2071

40. Herman T, Giladi N, Gurevich T et al (2005) Gait instability and fractal dynamics of older adults with a "cautious" gait: why do certain older adults walk fearfully? Gait Posture 21:178-185

41. Rende B, Ramsberger G, Miyake A (2002) Commonalities and differences in the working memory components underlying letter and category fluency tasks: a dual-task investigation. Neuropsychology 16:309-321 